\title{
Application of A Newly Developed Thermally Induced Volumetric Error Compensation Model in Improving Dimensional Accuracy of Parts
}

\author{
Yuxia Lu', M. N. Islam² \\ Department of Mechanical Engineering, Curtin University \\ Perth, WA 6845, Australia \\ 1'yuxia.lu@postgrad.curtin.edu.au, ${ }^{2}$ M.N.Islam@curtin.edu.au
}

Keywords: Thermally-induced volumetric error, error compensation, positioning error, dimensional accuracy

\begin{abstract}
The thermally induced volumetric error of a machine tool has been recognised as a major contributor to the dimensional and geometric errors of component parts produced by that tool. Therefore, considerable research has focused on ways to compensate for this type of error. The traditional model of compensation for the thermally induced volumetric error of a three-axis machine tool requires the measurement of 21 geometric error components and their variations with temperature, which is difficult and time-consuming to conduct. In our previous research, we developed a simplified and economical method of compensation of thermally induced volumetric error by modeling the positioning error as functions of ball-screw nut temperature and travel distance. This paper describes the application of this newly developed compensation model in improving the dimensional accuracy of parts. The model was applied to reduce the positioning error of a number of drilled holes. The results showed an average reduction of absolute and relative errors by $30.44 \mu \mathrm{m}$ and $77 \%$, respectively.
\end{abstract}

\section{Introduction}

The thermal condition of machine tools can profoundly affect the dimensional accuracy of manufactured parts; consequently, the reduction of its influence has become increasingly important to modern manufacturing. The measures proposed by the researchers to reduce the thermal influence fall into three categories [1]: (1) control of heat flow into the machine system, (2) redesign of the frame and scale to reduce their sensitivity to heat flow, and (3) compensation through controlled relative motions within the frame.

The most effective solution is design optimization of the machine tool. However, design methods alone are insufficient to completely avoid thermal displacements. Compensation techniques are necessary to increase the working accuracy, especially with regard to CNC machine tools [2].

Thermal errors can be predicted and compensated for by using mathematical models that apply data from a number of temperature points related to the increase of thermal errors and incorporating these errors within the general volumetric error model to form a synthesized model [3, 4]. However, this method encountered various difficulties when applied industrially, as it requires a high precision laser interferometer to measure the 21 geometric error components and their variations with temperature, as well as a large number of thermocouples to monitor temperature variations, which is costly and time-consuming.

We have developed a new method for calculating thermally induced volumetric error, in which the three axial positioning errors were modeled as functions of ball-screw nut temperature and travel distance [5,6]. This assumption was based on the fact that thermally induced positioning errors constitute over $80 \%$ of total volumetric error [5]. According to this method, a simple Laser Doppler Displacement Meter (LDDM) can be used as a substitute for the expensive laser interferometer; the large number of thermocouples necessary for inspecting the temperature variations can be reduced to three. 
The thermally induced volumetric error prediction accuracy of the model proposed above was verified by simulation results based on publically available data [7]. The results showed that only a negligible amount of dimensional accuracy would be sacrificed if compensated for by thermally induced positioning errors only [6]. In our recent research [8], we employed a LDDM to measure the thermally induced positioning errors produced in our 3-axis CNC vertical machining centre. The newly developed thermally induced volumetric error model [5, 6] was then modified based on the operating condition of the test machine [8]. The present paper demonstrates how the modified thermally induced volumetric error compensation model can be applied to improve the dimensional accuracy of parts.

\section{Volumetric Error Model}

Traditional Model. The geometric error of a three-axis machine tool consists of 21 error components, of which six are along each axis and three are squareness errors between axes. The six error components along each axis consist of three translation errors (one positioning error and two straightness errors) and three rotational errors (pitch, yaw, and roll). The error vector $V\left(V_{x}, V_{y}, V_{z}\right)$ of all 21 combined errors in the work volume (space) is defined as the volumetric error. The volumetric error at certain temperature state can be calculated by following the traditional model:

$$
\left\{\begin{aligned}
V_{x} & =\left(-Y_{d}\right) \cdot\left[\Sigma Z+\varepsilon_{z}(r)\right]+Z_{d} \cdot\left[\Sigma Y+\varepsilon_{y}(r)\right]+\Delta X \\
V_{y} & =\left(-Z_{d}\right) \cdot\left[\Sigma X+\varepsilon_{x}(r)\right]+X_{d} \cdot\left[\Sigma Z+\varepsilon_{z}(r)\right]+\Delta Y \\
V_{z} & =\left(-X_{d}\right) \cdot\left[\Sigma Y+\varepsilon_{y}(r)\right]+Y_{d} \cdot\left[\Sigma X+\varepsilon_{x}(r)\right]+\Delta Z \\
\Sigma X & =\varepsilon_{x}(x)+\varepsilon_{x}(y)+\varepsilon_{x}(z) \\
\Sigma Y & =\varepsilon_{y}(x)+\varepsilon_{y}(y)+\varepsilon_{y}(z) \\
\Sigma Z & =\varepsilon_{z}(x)+\varepsilon_{z}(y)+\varepsilon_{z}(z) \\
\Delta X & =\delta_{x}(x)+\delta_{x}(y)+\delta_{x}(z) \\
\Delta Y & =\delta_{y}(x)+\delta_{y}(y)+\delta_{y}(z) \\
\Delta Z & =\delta_{z}(x)+\delta_{z}(y)+\delta_{z}(z)
\end{aligned}\right.
$$

where, $P\left(X_{d}, Y_{d}, Z_{d}\right)$ represents the designed position; $\delta_{x}(x), \delta_{y}(y)$ and $\delta_{z}(z)$ are positioning errors; $\delta_{y}(x)$, $\delta_{z}(x), \delta_{x}(y), \delta_{z}(y), \delta_{x}(z)$, and $\delta_{y}(z)$ are straightness errors; $\varepsilon_{x}(x), \varepsilon_{y}(x), \varepsilon_{z}(x), \varepsilon_{y}(y), \varepsilon_{z}(y), \varepsilon_{x}(y), \varepsilon_{z}(z), \varepsilon_{y}(z)$, and $\varepsilon_{x}(z)$ are angular errors; $\varepsilon_{x}(r), \varepsilon_{y}(r)$ and $\varepsilon_{z}(r)$ are squareness errors.

Thermally Induced Volumetric Error Model. Based on the above mathematical model and the significant effect of the temperature on three positioning errors, we developed a new thermally induced volumetric error model [5]. This model assumes that thermally induced positioning errors are functions of the ball-screw nut temperature and the travel distance; the remaining 18 error components remain constant at their pre-calibrated cold start values.

The above model was further simplified by neglecting the angular errors and considering only the thermally induced positioning errors, in order to reduce the insignificant error components and simplify the calculation. The simulation results showed that only a negligible amount of dimensional accuracy could be sacrificed if compensating for the linear errors alone [6]. Details of these models [5, 6] are not included in the present paper due to lack of space.

The simplified model [6] was modified into following Eq. 2, based on the operating conditions of our test machine [8].

$$
\left\{\begin{array}{c}
V_{x}=E_{x}\left(x, T_{x n u t}\right)=E_{x}\left(x, T_{x 0}\right)+\beta_{x} \cdot \alpha \cdot x \cdot\left(T_{x n u t}-T_{x 0}\right) \\
V_{y}=E_{y}\left(y, T_{\text {ynut }}\right)=E_{y}\left(y, T_{y 0}\right)+\beta_{y} \cdot \alpha \cdot y \cdot\left(T_{\text {ynut }}-T_{y 0}\right) \\
V_{z}=E_{z}\left(z, T_{\text {znut }}\right)=E_{z}\left(z, T_{z 0}\right)+\beta_{z} \cdot \alpha \cdot z \cdot\left(T_{\text {znut }}-T_{z 0}\right)
\end{array}\right.
$$


where $x, y$ and $z$ is axial travel distance; $a$ is coefficient of linear thermal expansion; $\beta_{x}, \beta_{y}$ and $\beta_{z}$ are multiplication factors; $T_{x n u t}, T_{y n u t}$, and $T_{z n u t}$ are nut temperatures; $T_{x 0}, T_{y 0}$ and $T_{z 0}$ are nut temperatures at the cold start of the machine; $E_{x}\left(x, T_{x n u t}\right), E_{y}\left(y, T_{y n u t}\right)$, and $E_{z}\left(z, T_{z n u t}\right)$ are the positioning errors along axes that are functions of axial travel distance and nut temperature; $E_{x}\left(x, T_{x 0}\right), E_{y}\left(y, T_{y 0}\right)$, and $E_{z}\left(z, T_{z 0}\right)$ denote the original positioning errors, which can be measured before machine operation.

\section{Experimental Work}

Component Design. A simple test component (Figure 1) was designed to monitor the positioning accuracy of the centres of a number of drilled holes. A drilling operation was chosen because it is relatively simple to compensate for positioning errors of drilled hole centres. The datum planes $\mathrm{B}$ and $\mathrm{C}$ were machined before the drilling operation to assure the uniformity of reference surfaces for the co-ordinate measuring machine (CMM) inspection. A $12 \mathrm{~mm}$ diameter HSS drill mill combo cutter was employed to machine datum planes $\mathrm{B}$ and $\mathrm{C}$ and the five holes to reduce tool setup error. Sufficient space was left before hole H1 $(100 \mathrm{~mm})$ and after hole H5 (102 mm) for clamping the workpiece. Aluminum alloy 6061 was chosen as the work material.

Cutting Conditions. We performed all of the experiments under the same cutting conditions. Coolant was applied through the entire cutting process. The cutting parameters used for milling the datum planes were: cutting speed, $151 \mathrm{~m} / \mathrm{min}$; feed rate, $417 \mathrm{~mm} / \mathrm{min}$; and depth of cut, $0.5 \mathrm{~mm}$. The cutting parameters used for drilling the holes were: cutting speed, $162 \mathrm{~m} / \mathrm{min}$; and feed per revolution, $0.04 \mathrm{~mm} / \mathrm{rev}$.

Experimental Design. The aim was to evaluate the positioning accuracy of drilled hole centres with and without the application of the newly developed thermally induced volumetric error compensation model (Eq. 2). Therefore, we divided the experiments into two categories: the first was a preliminary test (PT) in which the workpiece was machined according to the designed dimension without any compensation; the second was a compensation test (CT), in which the test was conducted in exactly the same way as the preliminary test, but with compensated values. A total of 30 components were machined, 20 during the preliminary test and the remaining 10 during the compensation test (see Table 1 for details).

In the preliminary test, 10 of the 20 components were machined using identical cutting conditions and design dimensions as the cold stage $\left(22.5^{\circ} \mathrm{C}\right)$ of the machine tool. These components were marked as PTCS1-PTCS10 (PTCS; preliminary test in cold stage). The remaining 10 components were machined using the same cutting conditions and design dimensions as the thermal stable stage $\left(24.5{ }^{\circ} \mathrm{C}\right.$ ) of the machine tool. These components were marked as PTSS1-PTSS10 (PTSS; preliminary test at thermal stable stage).

In the compensation test, five of the 10 components were machined using identical cutting conditions, with compensated values for the dimensions at the cold stage $\left(22.5^{\circ} \mathrm{C}\right)$ of the machine tool, which were marked as CTCS1-5 (CTCS; compensation test in cold stage). The remaining five components were machined using identical cutting conditions and compensated dimensions for the thermal stable stage $\left(24.5{ }^{\circ} \mathrm{C}\right)$ of the machine tool, which were marked as CTSS1-5 (CTSS; compensation test at thermal stable stage).

The temperatures $\left(22.5^{\circ} \mathrm{C}\right.$ and $\left.24.5^{\circ} \mathrm{C}\right)$ refer to the nut temperature along the $x$-axis, which is the optimum temperature point when calculating the expansion of the lead screw used [5]. The reason we chose these two temperatures is that when the test machine is only used for a short period of time it works at $22.5^{\circ} \mathrm{C}$ (the room temperature in our laboratory is controlled at $21^{\circ} \mathrm{C}$ ); however, when the machine runs continually for normal machining over several hours, the $x$-axis nut temperature increases to $24.5^{\circ} \mathrm{C}$ [8].

Experimental Procedure. The experiments were conducted on a V30 CNC machining centre manufactured by Leadwell, Taiwan. A laser thermometer was used to measure the $x$-axis nut temperature. The measuring point was at the motor end and was as close as possible to the contact 
Table 1 Details of experiments

\begin{tabular}{|c|c|c|}
\hline Test & Test type & Temperature $\left[{ }^{\circ} \mathrm{C}\right]$ \\
\hline PTCS1-10 & Preliminary & 22.5 \\
\hline PTSS1-10 & Preliminary & 24.5 \\
\hline CTCS1-5 & Compensation & 22.5 \\
\hline CTSS1-5 & Compensation & 24.5 \\
\hline
\end{tabular}

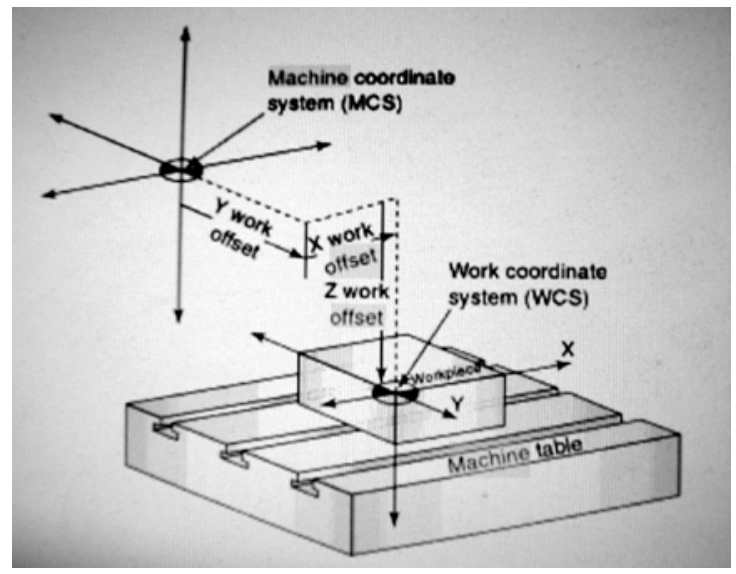

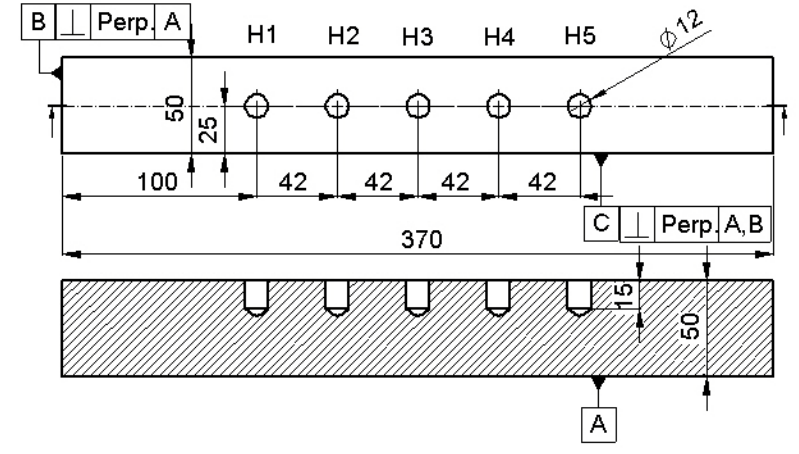

Fig.1 Test component

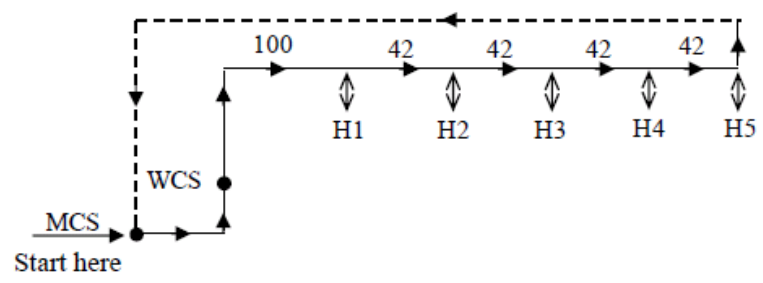

Fig. 3 Cutter path

Fig. 2 Relationship between machine coordinate system and work coordinate system

surface between the lead screw and the nut $[5,8]$. The details of the experimental procedure for the preliminary test and compensation test are as follows.

Preliminary Test: We machined the preliminary test components PTCS1-10 to examine the repeatability of the machine tool at its cold stage $\left(22.5{ }^{\circ} \mathrm{C}\right)$. We performed each experiment on a different day to ensure that all the tests were conducted at $22.5^{\circ} \mathrm{C}$. The blank material was initially clamped onto the machine table, according to the work coordinate system (WCS) and the machine was switched on (cold start). The relationship between the MCS (machine coordinate system) and the WCS is shown in Fig. 2. The blank material was secured at the same nominal position on the machine table for all of the conducted tests, and the $x$ work offset was $350 \mathrm{~mm}$ for the convenience of loading and unloading the workpiece. We then warmed the machine up to $22.5^{\circ} \mathrm{C}$ by moving the spindle head along the $x$-axis, which took approximately 20 minutes, after which we moved the machine back to the origin of the MCS. We then started the drilling process, which followed the cutter path shown in Fig. 3. The reason for moving the spindle head back to the origin of the MCS before starting the cutting process was to ensure that all the tests had exactly the same tool path.

We then machined the preliminary test components PTSS1-10 to assess the machine tool's repeatability at its thermal stable stage $\left(24.5^{\circ} \mathrm{C}\right)$. The procedure we followed was the same as that which we used for PTCS1-10, with an exception being that the machine was warmed up to $24.5^{\circ} \mathrm{C}$. This took approximately 100 minutes.

Compensation Test: The compensation test components CTCS1-5 and CTSS1-5 were machined at $22.5{ }^{\circ} \mathrm{C}$ and $24.5^{\circ} \mathrm{C}$, respectively, to assess the improvement of hole positioning accuracy after applying the proposed volumetric error compensation model. We followed the same procedures as for PTCS1-10 and PTSS1-10, respectively, with the exception being that we moved the drill tip according to the compensated values instead of the designed values. The calculation of compensation value is explained in the results and analysis section. 
The machined parts were inspected by a Discovery Model D-8 CMM, manufactured in Sheffield, UK. We used $4 \mathrm{~mm}$ diameter spherical probes, manufactured by Renishaw Electrical Ltd. We took measurements from datum planes $\mathrm{B}$ and $\mathrm{C}$, respectively, along the $x$-axis and $y$-axis and 10 points were probed at each height level (five levels per hole) to determine the centre position of the hole.

\section{Results and Analysis}

Analysis of Preliminary Test Results. The positioning errors of holes in the $x$-axis at the cold stage of the preliminary test (PTCS1-10) are shown in Fig. 4. The measurement refers to datum plane B, which is the origin of the WCS. A positioning error is defined as the measured position minus the designed position. As shown, all of the positioning errors were negative and the positioning errors for different workpieces had significant variations, although they were machined in similar thermal conditions $\left(22.5^{\circ} \mathrm{C}\right)$.

The positioning errors of holes in the $x$-axis at the thermal stable stage of the preliminary test (PTSS1-10) are shown in Fig. 5. As demonstrated, all the positioning errors remain negative; however, there is less variation between the components of the positioning error, compared to the workpiece machined at $22.5^{\circ} \mathrm{C}$ (Average $\pm 3 \sigma=-29.49 \pm 26.81 \mu \mathrm{m}$ for PTCS; $-39.41 \pm 11.35 \mu \mathrm{m}$ for PTSS). The comparison between these two figures reveals that the average positioning errors increased in a negative direction as the temperature increased to the thermal stable stage. Nevertheless, the components machined at the thermal stable stage produced improved repeatability of positioning accuracy compared to the workpiece machined at the cold stage. The explanation for the increase in positioning errors in a negative direction is outlined below.

With regard to the structure of the lead screw of the test machine we used ( $x$-axis), one end of the lead screw was connected to the driving motor and supported by a thrust bearing. This end can be considered as fixed. The other end, where the $x$-axis for the test machine originates, was supported by a ball-bearing and can be considered as the free end. Therefore, when the temperature increased, the origin of the MCS drifted in a negative direction (point P, as shown in Fig. 6), due to the thermal expansion of the lead screw. When the drill tip moved $350 \mathrm{~mm}$ from the origin of the MCS to reach the origin of the WCS as designed, the drill tip could not actually reach the precise origin of the WCS, because of the negative thermal drifts $\Delta \mathrm{x}$, even though the travel produces positive travelling errors $E_{m w}$ [8]. In fact, the real machining started from plane $B_{1}$ rather than datum plane B; i.e., the origin of the WCS had negative deviations caused by thermal drifts of the origin of the MCS. This is the primary reason behind significant negative positioning errors.

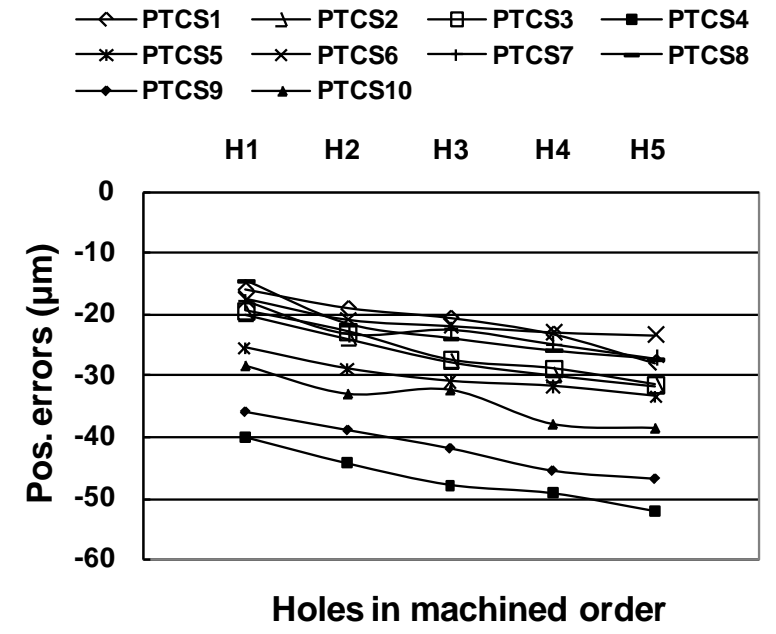

Fig. 4 Positioning errors of holes for preliminary test at cold stage (PTCS1-10)

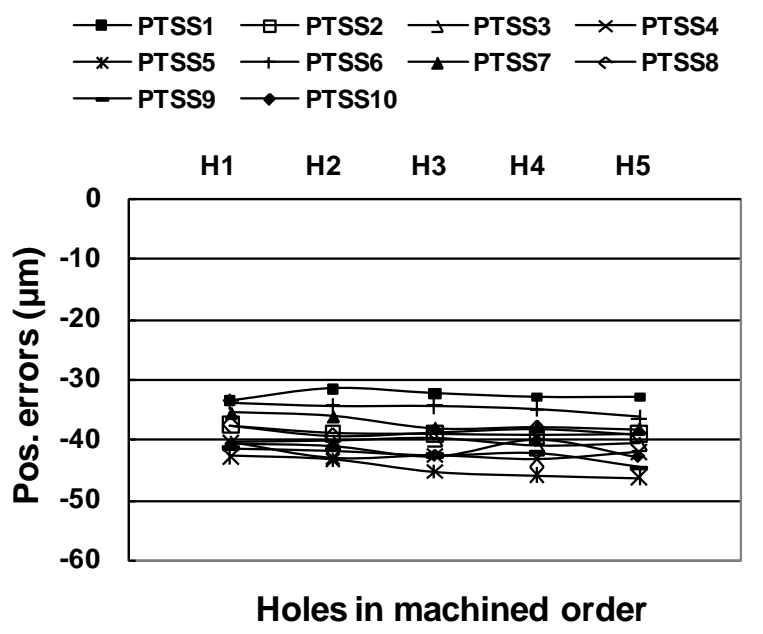

Fig. 5 Positioning errors of holes for preliminary test at thermal stable stage (PTSS1-10) 


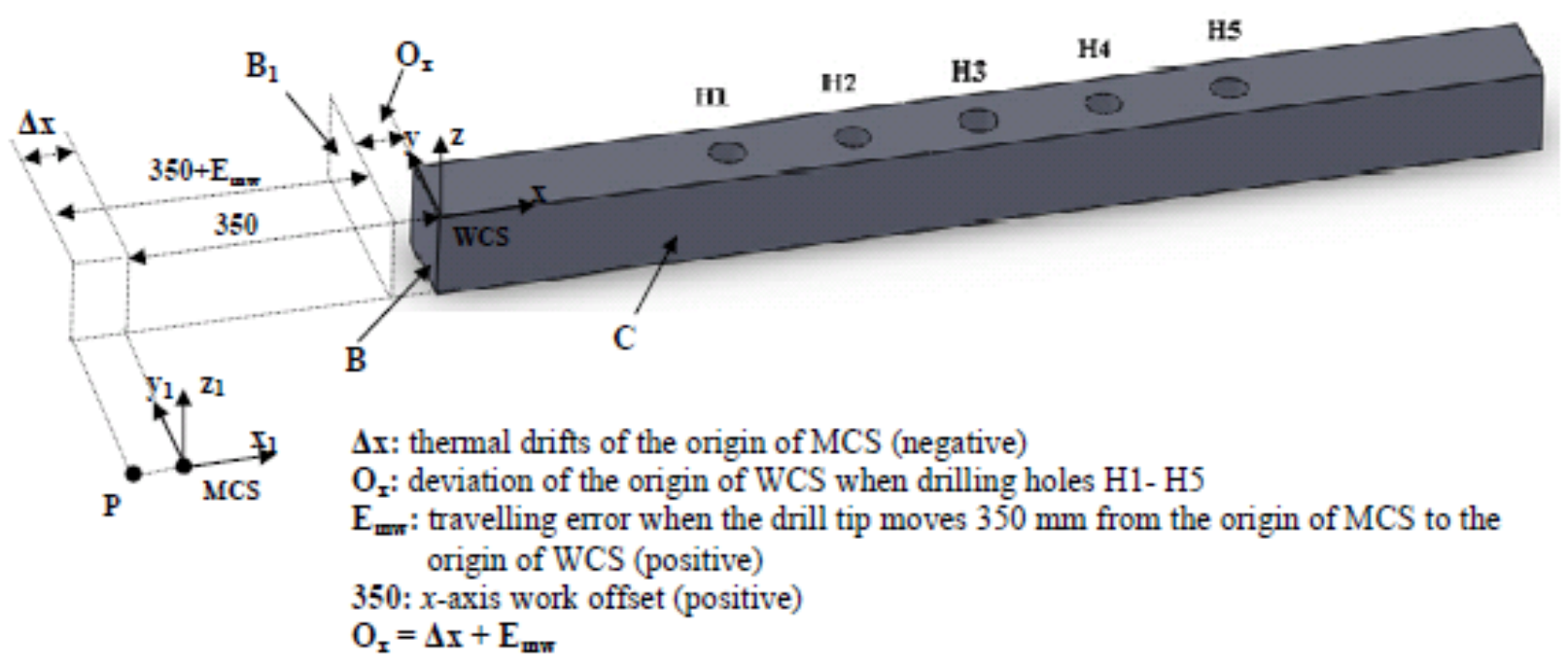

Fig. 6 Thermal drifts of the origin of the machine coordinate system

On the basis of the above analysis and the machining procedure performed in the workpiece (Fig. 1 ), the positioning error of the drilled hole (along the $x$-axis) calculation model can be modified from Eq. 2 to the following form:

$$
\left\{\begin{array}{l}
\operatorname{Err}_{x}\left(x, T_{\text {xnut }}\right)=O_{x}+\operatorname{RErr}_{x}\left(x, T_{\text {xnut }}\right) \\
\operatorname{RErr}_{x}\left(x, T_{\text {xnut }}\right)=\operatorname{RErr}_{x}\left(x, T_{x 0}\right)+\beta_{x} \cdot \alpha \cdot x \cdot\left(T_{x n u t}-T_{x 0}\right)
\end{array}\right.
$$

where,

$x$ : designed position on the $x$-axis with respect to the origin of the WCS;

$O_{x}$ : origin thermal deviations of the WCS, $O_{x}=\Delta x+E_{\text {mw }}$ (see Fig. 6);

$\Delta \mathrm{x}$ : thermal drifts of the origin of the MCS, its variations with the temperature can be measured and stored in machine's data bank;

$E_{\mathrm{mw}}$ : travelling error when the drill tip moves $350 \mathrm{~mm}$ from the origin of the MCS to the origin of WCS, its value can be determined from Eq. 2, as has been verified in our previous research [8];

$\operatorname{RErr}_{x}\left(x, T_{x n u t}\right)$ : relative positioning errors when the machine travels from the origin of the WCS to the target position at temperature $T_{x n u t}$;

$\operatorname{RErr}_{x}\left(x, T_{x 0}\right)$ : relative positioning errors when the machine travels from the origin of the WCS to the target position at the cold start $\left(T_{x 0}\right)$ of the machine, its value can be determined from the measured results [8];

$\beta_{x}$ : multiplication factor in the $x$-axis, its value refers to our previous research [8].

The compensation value is calculated by the nominal position of the hole minus the positioning error. For example, the nominal position of the first hole $\mathrm{H} 1$ is $100 \mathrm{~mm}$, and this will be substituted with a compensation value $x_{\text {comp. }}$. when drilling the first hole $\mathrm{H} 1$ in the compensation test (CTCS and

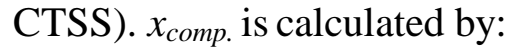

$$
x_{\text {comp. }}=100-\operatorname{Err}_{x}\left(x, T_{\text {xnut }}\right)
$$

where, $\operatorname{Err}_{x}\left(x, T_{\text {xnut }}\right)$ can be calculated from Eq. 3.

Analysis of Compensation Test Results. As explained above, the components machined in the preliminary test stage (PTCS and PTSS) are the components without compensation, while the components machined in the compensation test stage (CTCS and CTSS) are the components with compensation. The comparisons of positioning errors between machining with and without compensation, and their distributions, are summarised in Fig. 7 and Fig. 8. As shown, after we applied the proposed thermally induced volumetric error compensation model, the positioning 
accuracy of machined holes improved significantly; however, there was little variability in the repeatability of machining and the error distribution range.

Although the amount of negative positioning errors was reduced significantly after application of the thermally induced volumetric error model, some negative errors still remained. This is because we did not consider some of the factors that have important effects on the positioning accuracy of the drill tip, such as spindle tilt caused by continuous rotating and column bending, workpiece thermal deformation, and natural variation of the drilling process, during the course of this research.

Despite these limitations, the proposed thermally induced volumetric error compensation method significantly improved the positioning accuracy of drilled hole centres. The reduction of positioning errors by compensation is summarised in Table 2 . The average absolute reduction of the positioning

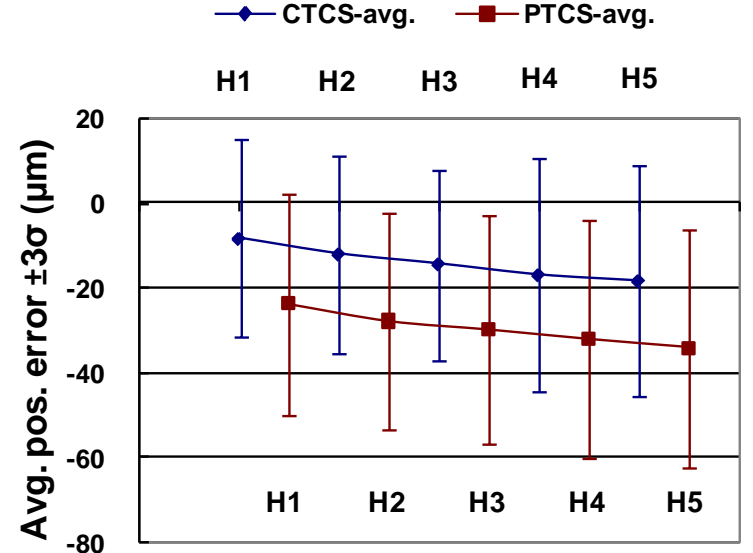

Holes in machined order

Fig.7 Comparison of average positioning errors $( \pm 3 \sigma)$ between machining with compensation (CTCS) and without compensation (PTCS) at cold stage

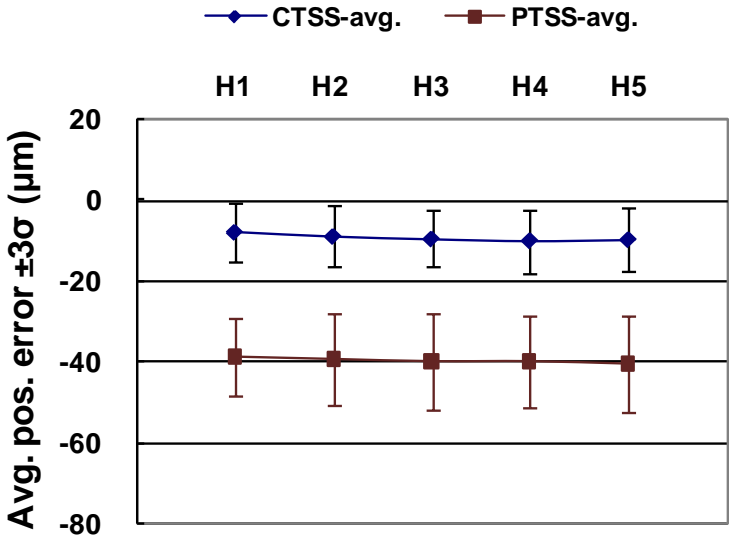

Holes in machined order

Fig.8 Comparison of average positioning errors $( \pm 3 \sigma)$ between machining with compensation (CTSS) and without compensation (PTSS) at thermal stable stage

Table 2 Positioning error reduction after compensation

\begin{tabular}{|c|c|c|c|c|c|}
\hline \multicolumn{2}{|c|}{ Holes } & $\begin{array}{c}\text { Without } \\
\text { compensation }\end{array}$ & $\begin{array}{c}\text { With } \\
\text { Compensation }\end{array}$ & $\begin{array}{l}\text { Absolute } \\
\text { Reduction }\end{array}$ & $\begin{array}{l}\text { Relative } \\
\text { Reduction }\end{array}$ \\
\hline \multirow{6}{*}{$\begin{array}{c}\text { 22.5-avg.* } \\
\text { [--CS] }\end{array}$} & 1 & -23.61 & -8.32 & 15.29 & 65 \\
\hline & 2 & -27.76 & -12.00 & 15.76 & 57 \\
\hline & 3 & -29.82 & -14.35 & 15.47 & 52 \\
\hline & 4 & -32.13 & -16.93 & 15.19 & 47 \\
\hline & 5 & -34.11 & -18.35 & 15.77 & 46 \\
\hline & Average** $^{* *}$ & -29.49 & -13.99 & 15.50 & 53 \\
\hline \multirow{6}{*}{$\begin{array}{c}\text { 24.5-avg* } \\
\text { [--SS] }\end{array}$} & 1 & -38.42 & -7.60 & 30.82 & 80 \\
\hline & 2 & -39.04 & -8.68 & 30.36 & 78 \\
\hline & 3 & -39.64 & -9.32 & 30.32 & 76 \\
\hline & 4 & -39.64 & -9.76 & 29.88 & 75 \\
\hline & 5 & -40.30 & -9.50 & 30.80 & 76 \\
\hline & Average** & -39.41 & -8.97 & 30.44 & 77 \\
\hline \multicolumn{6}{|c|}{$\begin{array}{l}+ \text { Absolute reduction }=\text { abs (error without compensation) }- \text { abs (error with compensation) } \\
++ \text { Relative reduction }=100 * \text { absolute reduction/abs(error without compensation) } \\
* \text { Average of holes in different workpiece and the same } x \text { position, such as H1 in PTCS1-10 } \\
* * \text { Average of all holes in the same type of test, such as all holes in PTCS }\end{array}$} \\
\hline
\end{tabular}


errors of drilled holes was $15.50 \mu \mathrm{m}$ at the cold stage $\left(22.5^{\circ} \mathrm{C}\right)$ and $30.44 \mu \mathrm{m}$ at the thermal stable stage $\left(24.5^{\circ} \mathrm{C}\right)$, while the average relative reduction ratio of these positioning errors was $53 \%$ at the cold stage $\left(22.5^{\circ} \mathrm{C}\right)$ and $77 \%$ at the thermal stable stage $\left(24.5^{\circ} \mathrm{C}\right)$.

\section{Conclusion}

Based on our experiments and subsequent analysis, we have drawn the following conclusions:

- Thermally induced positioning errors include two key components: thermally induced origin drifts and relative or travelling positioning error. Although a travelling positioning error for the test machine is positive and proportional to the travel distance [8], the test machine used for the present study had a considerable negative origin thermal drift, which significantly affected the dimensional accuracy of the machined workpiece.

- For a small-sized workpiece, the thermal origin drifts could dominate the positioning error, so it is essential to avoid, or compensate for, the origin thermal drifts when planning the machining procedure.

- A machine tool produces reliable high dimensional replication at the thermal stable stage, so this stage is the optimal stage at which to conduct thermal error compensation.

- After we applied the proposed thermally induced volumetric error compensation model, the positioning accuracy of drilled holes improved significantly. The absolute reduction of the positioning errors of drilled holes was an average $30.44 \mu \mathrm{m}$ at the thermal stable stage, while the average relative reduction ratio of these errors was $77 \%$.

\section{References}

[1] J. Bryan: International status of thermal error research. Annals of the CIRP, 39 (2), (1990), pp.645-656.

[2] G. Spur, E. Hoffmann, Z. Paluncic, K. Benzinger and H. Nymoen: Thermal behaviour optimisation of machine tools. Annals of the CIRP, 37 (1), (1988), pp. 401-405.

[3] R. Ramesh, M.A. Mannan and A.N. Poo: Error compensation in machine tools -- a review: Part II: Thermal errors. International Journal of Machine Tools and Manufacture, 40(9), (2000), pp.1257-1284.

[4] J.-H. Lee and S.-H. Yang: Statistical optimization and assessment of a thermal error model for CNC machine tools. International Journal of Machine Tools and Manufacture, 42, (2002), pp.147-155.

[5] Y. Lu and M.N. Islam: A new approach to thermally induced volumetric error compensation. International Journal of Advanced Manufacturing Technology, (2011) DOI 10.1007/s00170-011-3849-6.

[6] Y. Lu and M.N. Islam: A simplified method for thermally induced volumetric error compensation. International Review of Mechanical Engineering (IREME), 5(5), (2011).

[7] R. Venugopal: Thermal effects on the accuracy of numerically controlled machine tools. (PhD Thesis, Purdue University, 1985).

[8] Y. Lu and M.N. Islam: Prediction and analysis of thermal effects on dimensional accuracy of machined parts. Proc. of the International Conf. on Mechanical Engineering and Mechatronics, Ottawa, Ontario, Canada, 15-17 August 2012, Paper No 31, pp. 1-8.

[9] P.J. Hoffman, E.S. Hopewell, B. Janes, K.M. Shart, Jr.: Precision machining technology (Delmar Cengage Learning, USA). 